\title{
Planeamiento Integrado de la Expansión Generación- Transmisión utilizando los Índices Nodales Ponderados de Alivio de Carga en Transmisión (WTLR)
}

\author{
Sergio D. Saldarriaga-Zuluaga, Jesús M. López-Lezama, Nicolás Muñoz-Galeano \\ Fac. de Ingeniería, Dpto. Ing. Eléctrica, Grupo de Manejo Eficiente de la Energía - GIMEL, Universidad de \\ Antioquia, Calle 67 No. 53-108, Oficina 19-437, Medellín, Colombia \\ (e-mail: sergio.saldarriaga@udea.edu.co,jmaria.lopez@udea.edu.co,nicolas.munoz@udea.edu.co)
}

Recibido Abr. 4, 2016; Aceptado May. 24, 2016; Versión final Jun. 17, 2016, Publicado Dic. 2016

\begin{abstract}
Resumen
Este artículo presenta un modelo para el Planeamiento Integrado de la Expansión en Generación y Transmisión (PIEGT). La contribución principal de este artículo consiste en la utilización de los índices nodales de Alivio de Carga en Transmisión Ponderada (WTLR, Weighted Transmission Loading Relief) para la identificación de nuevos candidatos de expansión (líneas y generadores). Los índices WTLR están dados en función de los factores de distribución de potencia y permiten medir la severidad de las sobrecargas, tanto en operación normal, como en contingencia (criterio $\mathrm{N}-1$ ). La aplicabilidad del modelo propuesto se evaluó en el sistema de prueba IEEE RTS de 24 barras. EI PIEGT se solucionó mediante la técnica de Algoritmos Genéticos Clasificados No-dominados II (NSGA-II, Non-dominated Sorting Genetic Algorithm II) teniendo como objetivos la minimización de costos y la maximización de la seguridad del sistema. La inclusión de generación como alternativa en la expansión reduce el número de líneas de transmisión necesarias del plan de expansión, especialmente cuando se tienen en cuenta criterios de seguridad.
\end{abstract}

Palabras clave: planeamiento integrado; generación y transmisión; factores de distribución; optimización multiobjetivo; NSGA-II

\section{Integrated Transmission and Generation Expansion Planning using Weighted Transmission Loading Relief Nodal Indexes (WTLR)}

\begin{abstract}
This paper presents a model for the integrated Transmission and Generation Expansion Planning (TGEP). The main contribution of this paper lays in the use of the WTLR indexes (Weighted Transmission Loading Relief) for identifying new expansion candidates (lines and generators). The WTLR indexes are given as function of the distribution power factors and allow measuring the severity of overloads in normal conditions and under contingency ( $\mathrm{N}-1$ criterion). The applicability of the proposed model was tested in the IEEE RTS 24 bus power system. The TGEP was solved by means of the NSGA-II (Non-dominated Sorting Genetic Algorithm II) with the aim of minimizing costs and maximizing system security. The inclusion of generators as expansion alternatives reduces the number of necessary transmission lines in the expansion plan, especially when security criteria are taken into account.
\end{abstract}

Keywords: integrated generation; transmission planning; distribution factors; multiobjective optimization; NSGA-II 


\section{INTRODUCCIÓN}

El planeamiento de la expansión de los sistemas eléctricos de potencia tiene como objetivo suplir las necesidades de la demanda eléctrica que tendrá determinada región en el futuro. La atención de la demanda en el horizonte de planeación debe hacerse de manera económica, cumpliendo con criterios de confiabilidad y seguridad. En la búsqueda de soluciones para el planeamiento de la expansión, las variables más relevantes que inciden en la toma de decisiones son: proyección de la demanda en el horizonte de planeación, hidrología, costo de combustibles, fuentes de recursos energéticos, políticas e impactos ambientales.

La alta complejidad del problema de planeación de la expansión de los sistemas de potencia y la liberación de los mercados eléctricos han contribuido a que tradicionalmente el problema del planeamiento de la expansión se realice de forma separada para la generación y para la transmisión. En la literatura especializada se encuentran investigaciones que se centran únicamente en el Planeamiento de la Expansión de la Generación (PEG), por mencionar algunos, (Phupha et al., 2012) y (Hemmati et al., 2013) recopilan y describen las principales metodologías del PEG. De igual forma, existen estudios que solo se centran en el Planeamiento de la Expansión de Transmisión (PET) (Hemmati y Hooshmand, 2013; Quintero et al., 2014; Romero et al., 2002).

Recientemente, el problema del Planeamiento Integrado de la Expansión en Generación y Transmisión (PIEGT) se ha propuesto como alternativa para mejorar la solución del problema de la expansión. De acuerdo con (Jenabi et al., 2013), las nuevas propuestas de modelos de PIEGT se dividen principalmente en dos enfoques: 1) Modelo integrado de planeación generación-trasmisión. En este enfoque se asume que una empresa regulada y centralizada es la encargada de definir el desarrollo de todo el sistema, es decir, que plantas generadoras y que líneas de trasmisión se requieren en el sistema. El objetivo es maximizar el beneficio social y reducir los costos de inversión y operación. Modelos de este tipo se presentan en (Sharan y Balasubramanian, 2012) y (Ma y Zhou, 2012). 2) Modelo anticipativo de planeación generación-trasmisión. Este enfoque se origina en el hecho de que el agente transmisor tiene que anticipar los resultados del mercado al momento de decidir sobre la inversión en transmisión, adelantándose al portafolio de inversión del agente generador. Las decisiones en el planeamiento de la generación son hechas por empresas rivales en un mercado eléctrico competitivo, lo que implica que puede formularse como un problema de optimización que maximiza el bienestar social o la ganancia del productor. Modelos de este tipo se presentan en (Motamedi et al, 2010), (Jin y Ryan, 2014) y (Pozo et al., 2013).

En la literatura técnica se han propuesto diferentes métodos de solución para abordar el PIEGT. De forma general, se puede decir que estos métodos se dividen en tres grupos: técnicas de programación matemática, técnicas heurísticas y técnicas metaheurísticas. Ejemplos de aplicación de estas técnicas al PIEGT pueden ser consultadas en (Sharan y Balasubramanian, 2012), (Rouhani et al., 2014) y (Javadi et al., 2013), respectivamente.

Sin bien en la literatura se encuentran trabajos que abordan de forma conjunta el planeamiento integrado de la expansión en generación-trasmisión existe la necesidad de explorar nuevos modelos que permitan mejorar la toma de decisiones. Por lo que, en este artículo se propone un modelo de PIEGT que presenta como novedad la utilización de los índices WTLR definidos en función de los factores de distribución de potencia. La utilización de los índices WTLR permite identificar los nodos del sistema en los que es conveniente instalar generación y ramas de la red donde es conveniente instalar nuevas líneas de trasmisión. Adicionalmente, los WTLR permiten medir la severidad de las sobrecargas del sistema en estado normal y bajo contingencia (criterio $\mathrm{N}$-1). Por otro lado, el modelo propuesto utiliza los factores ISF (Injection Shift Factors), PTDF (Power Transfer Distribution Factors) y LODF (Line Outage Distribution Factors) para realizar el cálculo de las contingencias del sistema de una forma rápida y simple. El modelo propuesto es solucionado por medio de un algoritmo genético multiobjetivo NSGA-II, para el cual se considera como funciones objetivo: la minimización del costo de inversión y la minimización de los índices nodales WTLR (maximización de la seguridad).

\section{FORMULACIÓN MATEMÁTICA}

La formulación matemática del PIEGT se presenta en las ecuaciones (1)-(21). Se propone un modelo multiobjetivo que busca minimizar el costo de inversión de la expansión y a la vez minimizar el valor absoluto del máximo de los índices nodales WTLR. Para el cálculo de los factores de distribución de potencia se utiliza la formulación propuesta por Güler et al. (2007). 
Min: $f 1=\sum_{i j \in \Omega_{l n}} c_{l} w_{l}+\sum_{k \in \Omega_{g n}} c_{k} z_{k}+\sum_{k \in \Omega_{g n}} c o_{k} g_{k i} z_{k}+\sum_{k \in \Omega_{g}} c o_{k} g_{k i}+\sum_{i \in \Omega_{b}} D N A_{i} C_{D N A i}$

Min: $f 2=\operatorname{MAX}_{i \in \Omega_{b}} \quad\left|W T L R_{i}\right|$

Sujeto a:

$W T L R_{i}=\frac{\text { Nviol }}{\text { sobrecarga }}\left(\sum_{\text {sys }} I S F_{l}^{i} P C O_{l}+\sum_{l \in\left(\Omega_{l} \cup \Omega_{l n}\right)} \sum_{l n} I S F_{l, c}^{i} P C \Omega_{l, c}\right) \forall i \in \Omega_{b}$

$P C O_{l}=\sum_{l \in\left(\Omega_{l} \cup \Omega_{l n}\right)}\left(f_{l i j}-\bar{f}_{l}\right) \leftrightarrow f_{l i j}>\bar{f}_{l}$

$\forall l \in\left(\Omega_{l} \cup \Omega_{l n}\right)$

$P C O_{l}=0 \leftrightarrow f_{l i j} \leq \bar{f}_{l}$

$\forall l \in\left(\Omega_{l} \cup \Omega_{l n}\right)$

$P C O_{l, c}=\sum_{l \in\left(\Omega_{l} \cup \Omega_{l n}\right)}\left(f_{l i j, c}-\bar{f}_{l}\right) \leftrightarrow f_{l i j, c}>1.2 * \bar{f}_{l}$

$\forall l \in\left(\Omega_{l} \cup \Omega_{l n}\right), \forall c \in\left(\Omega_{c}\right)$

$P C O_{l, c}=0 \leftrightarrow f_{l i j, c} \leq 1.2 * \bar{f}_{l}$

$\forall l \in\left(\Omega_{l} \cup \Omega_{l n}\right), \forall c \in\left(\Omega_{c}\right)$

$f_{l i j, c}=f_{l i j}+L O D F_{l, c} f_{c}$

$\forall l \in\left(\Omega_{l} \cup \Omega_{l n}\right), \forall c \in\left(\Omega_{c}\right)$

$I S F_{l, c}^{i}=I S F_{l}^{i}+L O D F_{l, c} I S F_{c}^{i}$

$\forall l \in\left(\Omega_{l} \cup \Omega_{l n}\right), \forall c \in\left(\Omega_{c}\right)$

sobrecarga $_{\text {sys }}=\sum_{l \in\left(\Omega_{l} \cup \Omega_{l n}\right)} P C O_{l}+\sum_{c \in \Omega_{c}} \sum_{l \in\left(\Omega_{l} \cup \Omega_{l n}\right)} P C O_{l, c}$

$\forall l \in\left(\Omega_{l} \cup \Omega_{l n}\right)$

$\left(\sum_{l \in \Omega_{l}} f_{l j i}+\sum_{l \in \Omega_{l n}} f_{l j i} w_{l}\right)-\left(\sum_{l \in \Omega_{l}} f_{l i j}+\sum_{l \in \Omega_{l n}} f_{l i j} w_{l}\right)+\sum_{l \in \Omega_{g n}} g_{k i} z_{k}+\sum_{l \in \Omega_{g}} g_{k i}=d_{i}$

$\forall i \in \Omega_{b}$

$f_{l i j}=S_{\text {base }} \frac{\left(\theta_{i}-\theta_{j}\right)}{x_{l}^{p u}}$

$\forall l \in \Omega_{l}$

$f_{l i j}=0 \leftrightarrow w_{l}=0$

$\forall l \in \Omega_{l n}$

$f_{l i j}=S_{\text {base }} \frac{\left(\theta_{i}-\theta_{j}\right)}{x_{l}^{p u}} \leftrightarrow w_{l}=1$

$\forall l \in \Omega_{l n}$

$0 \leq g_{k i} \leq \bar{g}_{k}$

$\forall k \in\left(\Omega_{g}\right)$

$g_{k i}=0 \leftrightarrow z_{k}=0$

$\forall k \in\left(\Omega_{g n}\right)$

$0 \leq g_{k i} \leq \bar{g}_{k} \leftrightarrow z_{k}=1$

$\forall k \in\left(\Omega_{g n}\right)$

$-\bar{\theta} \leq \theta_{i} \leq \bar{\theta}$

$\forall i \in \Omega_{b}$

$w_{l}$ binario

$\forall l \in \Omega_{l n}$,

$z_{k}$ binario

$\forall k \in \Omega_{g n}$,

$\theta_{i}=0$

$\forall i \in \Omega_{b} / i=r e f$ 
La notación para conjuntos, parámetros y variables del modelo se presentan al final del artículo. La primera función objetivo, dada por la ecuación (1), consiste en la minimización de los costos de inversión y operación. Los primeros dos términos representan los costos de inversión para nuevas líneas y generadores, respectivamente. En este caso las variables binarias $\mathrm{z}_{\mathrm{k}} \mathrm{y} \mathrm{w}_{\mathrm{l}}$ se usan para indicar si existen nuevos generadores y líneas respectivamente. Por lo tanto, para un candidato de solución estas variables son parámetros con los que posteriormente se evalúa la función objetivo. El tercer y cuarto término indican el costo de operación para generadores nuevos y existentes, respectivamente. Finalmente, el último término representa el costo de la demanda no atendida. La segunda función objetivo, expresada en la ecuación (2), representa la minimización de los índices nodales WTRL, los cuales están definidos en la ecuación (3). En este caso los WTLR se calculan de forma exógena una vez se tengan definidas las variables $z_{k}$ y $w_{1}$ que representan un candidato de expansión. Los términos para el cálculo de estos índices están dados por las ecuaciones (4) a (7).

Los índices nodales WTLR fueron propuestos inicialmente en (Grijalva y Visnesky, 2006) y pueden ser utilizados para verificar si la instalación de nuevos generadores o líneas de transmisión contribuyen a mejorar la seguridad del sistema. Estos índices indican el cambio aproximado en la sobrecarga total del sistema (en condiciones normales y bajo contingencia) que se obtendría con una inyección adicional de 1 MW en una barra en particular. Los índices WTLR pueden tomar valores negativos o positivos. Los extremos receptores de elementos sobrecargados presentan índices WTLR negativos, lo que indica que inyectar potencia en estos nodos produce flujos contrarios que alivian la sobrecarga. Los extremos emisores de elementos sobrecargados presentan índices positivos, lo que indica que inyectar potencia en estos nodos empeoraría la sobrecarga. Para reducir las sobrecargas en condición normal y bajo contingencia, se deben instalar nuevos elementos (líneas y generadores) de tal forma que se reduzca la magnitud de los índices WTLR. Es decir, si estos índices son cero significa que no hay sobrecargas en operación normal ni bajo contingencias.

Las ecuaciones (4) y (5) representan los límites de sobrecarga en condición normal de operación para las líneas. Las restricciones (6) y (7) modelan los límites de flujo en cada línea, bajo contingencia. En este caso, se permiten sobrecargas de hasta el $120 \%$ del límite máximo de capacidad de las líneas. La restricción (8) permite realizar el cálculo del flujo post-contingencia de cada línea ante cada contingencia mediante los índices LODF. Estos índices representan la sensibilidad del cambio del flujo de potencia en cada línea para cada contingencia. La restricción (9) representa el ISF de cada línea con respecto a cada nodo ante cada contingencia. La restricción (10) es la ecuación para el cálculo de la sobrecarga total del sistema. La restricción (11) representa la ecuación de balance de potencias nodal. La restricción dada por la ecuación (12) modela los flujos de carga para las líneas existentes, mientras las restricciones (13) y (14) representan los flujos de carga para las líneas candidatas de expansión. La restricción dada por la ecuación (15) limita la generación máxima de cada generador existente. Las restricciones (16) y (17) limitan la generación máxima de cada generador nuevo. La restricción (18) representa los límites máximos de los ángulos de las barras. Las restricciones (19) y (20) consideran la naturaleza binaria de las variables de decisión para la inversión en líneas y generadores, respectivamente. Finalmente, la restricción (21) indica que el ángulo de la barra de referencia debe ser cero.

\section{ALGORITMO NSGA-II (Non-dominated Sorting Genetic Algorithm II)}

Para abordar el modelo propuesto en (1)-(21) se ha optado por la aplicación de una técnica metaheurística multiobjetivo. El algoritmo implementado en este trabajo fue propuesto inicialmente en (Srinivas y Deb, 1994) y se denominó NSGA; posteriormente, fue mejorado por (Deb et al., 2002), recibiendo el nombre NSGA-II. La optimización multiobjetivo ha sido utilizada para resolver los problemas PEG y PET de forma independiente. Un ejemplo de aplicación de estas técnicas, para el PEG, se presenta en (Aghaei et al., 2013); por otro lado, en (Ugranli y Karatepe, 2015) se presenta un algoritmo multiobjetivo para el PET. El NSGA-II también ha mostrado ser una herramienta efectiva para abordar modelos multiobjetivo en problemas diferentes al planeamiento de la expansión como se indica en (Rojas-Rios et al., 2016) y (Suarez et al., 2013). El diagrama de flujo del algoritmo implementado se presenta en la Fig.1.

\section{Población inicial}

La población inicial se genera de forma aleatoria. Cada individuo corresponde a un vector compuesto por unos y ceros, donde cada elemento del vector corresponde a una línea candidata o a un generador candidato de expansión. La longitud del vector corresponde al número de líneas y generadores candidatos. Si el valor de la posición del vector es cero significa que el elemento correspondiente no fue seleccionado como candidato para la expansión. Si el valor de la posición es uno significa que el elemento correspondiente fue seleccionado como candidato para la expansión. 


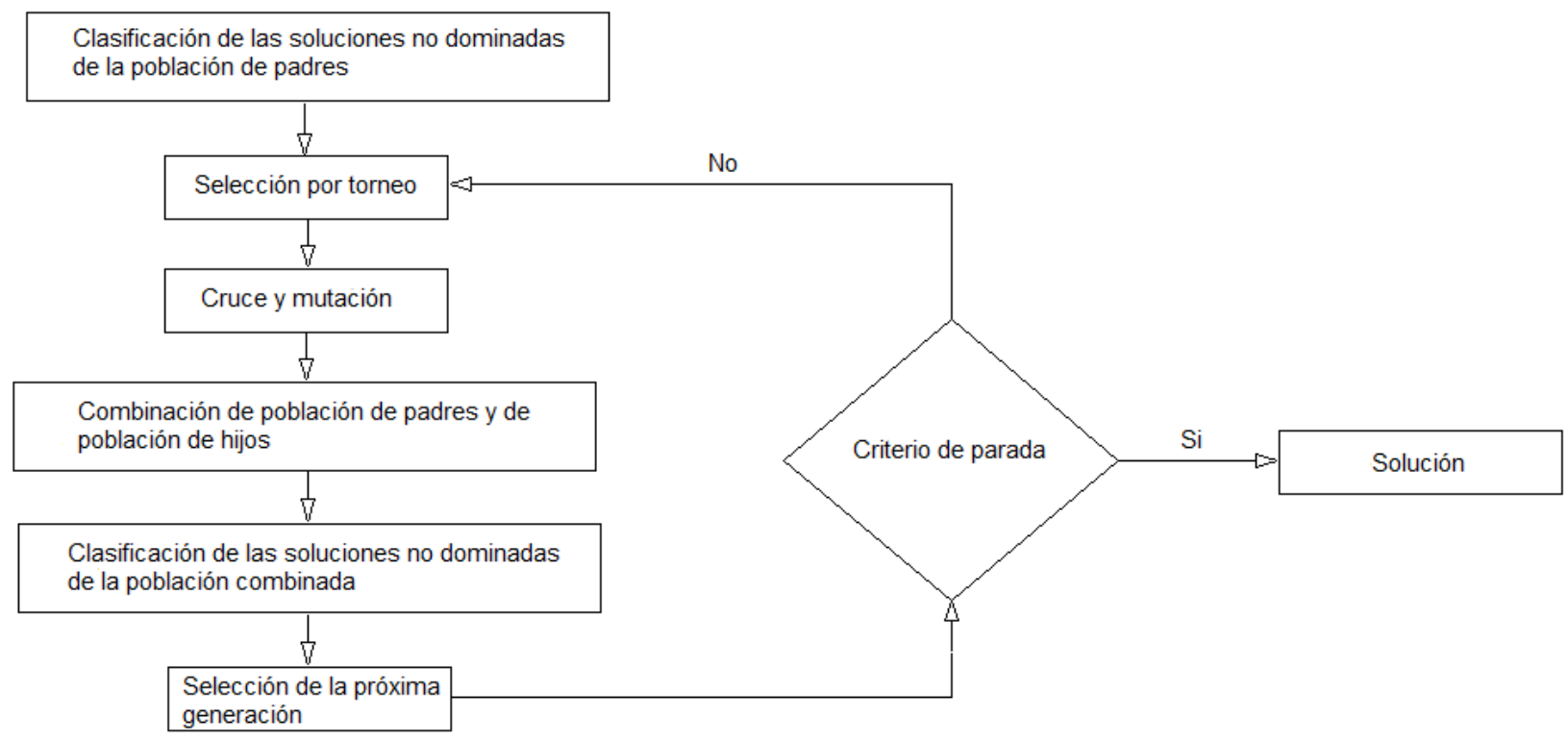

Fig.1: Diagrama de flujo del algoritmo NGSA-II

\section{Clasificación de las soluciones no dominadas de la población de padres}

Una vez se evalúan las funciones objetivo de los candidatos de expansión, se aplica el concepto de dominancia para clasificar las soluciones. Se dice que una solución $x$ domina a una solución $y$ cuando se cumple que la solución $x$ no es peor que la solución $y$ en ninguna de las funciones objetivo, además, la solución $x$ es mejor que la solución $y$ en al menos una de las funciones objetivo. La población se clasifica con base en la no dominancia en diferentes frentes de Pareto.

\section{Selección por torneo}

Una vez realizada la clasificación basada en la no dominancia y el cálculo de distancias entre soluciones, se debe escoger algunos individuos entre los mejores para generar nuevas posibles soluciones. En este caso, se implementa la sección por torneo binario que consiste en seleccionar aleatoriamente dos parejas de individuos y escoger el individuo con el mejor desempeño. Se realizan tantos torneos como número de individuos haya. Los mejores individuos (en este caso los mejores planes de expansión) pasan a la siguiente etapa de cruce y mutación.

\section{Cruce y mutación}

El cruce o recombinación es la parte del algoritmo en el que los padres intercambian su material genético para generar nuevos individuos (candidatos de expansión). La mutación consiste en alterar uno de los individuos, lo que permite, eventualmente, escapar de óptimos locales incorporando diversificación en la población. En este caso, el cruce y la mutación se dan con una probabilidad dada. El cruce se realiza seleccionando aleatoriamente dos padres de los ganadores del torneo y cruzando sus bits, esto genera individuos que comparten parte del material genético de los padres (hijos). En la mutación se selecciona aleatoriamente uno de los padres ganadores del torneo para posteriormente mutar, con cierta probabilidad, algunos de sus bits. Una vez se termina el proceso de cruce y mutación se procede a combinar la población de hijos y padres.

\section{Clasificación de las soluciones no dominadas de la población combinada}

La población combinada también se clasifica con base en la no dominancia de los diferentes frentes de Pareto. A cada población se le asigna un rango a su nivel de no dominación y se calcula la distancia de esta solución respecto a otras soluciones.

\section{Selección de la próxima generación}

Para la selección de la nueva población se utiliza la clasificación basada en la no dominancia. La nueva generación empieza a ser formada con el mejor frente no dominado de acuerdo a su rango, seguido por el segundo frente no dominado y así sucesivamente hasta terminar de seleccionar la mitad de la población 
combinada de padres e hijos, la otra mitad debe ser descartada. Si el rango de dos individuos es igual, se usa la distancia de este individuo respecto a otros individuos como criterio de selección, se prefiere la solución que este más alejada de las otras soluciones.

\section{Criterio de parada}

Existen básicamente dos criterios de parada: el número total de iteraciones o un número máximo de iteraciones sin que se haya encontrado una mejora en los individuos. Si se cumple cualquiera de estos dos criterios el algoritmo se detiene.

\section{PRUEBAS Y RESULTADOS}

Para verificar la validez del modelo propuesto se consideró el sistema de prueba IEEE RTS-24 planteado en (Grigg et al., 1999). Este sistema cuenta con 24 barras, 38 líneas, 32 generadores y 17 cargas que suman un valor de demanda de $2850 \mathrm{MW}$. Se desea encontrar la inversión óptima del mínimo costo para su expansión cumpliendo con criterios de seguridad. El valor de la carga futura es de 8550 MW, lo que representa tres veces el valor de la demanda actual. Las pruebas se realizaron con un computador de procesador AMD E-350 y 4GW de memoria RAM.

Para realizar las pruebas del modelo propuesto, se consideraron los corredores existentes y 7 corredores nuevos como candidatas de expansión en transmisión. En este sistema se permite adicionar hasta 2 líneas por cada corredor. Los parámetros de los corredores considerados como candidatos pueden ser consultados en (Romero et al., 2005). Además, para cada nodo del sistema se consideró la posibilidad de instalar tres tipos de generadores como candidatos de expansión en generación. El generador tipo 1 tiene una potencia máxima de $10 \mathrm{MW}$, el tipo $220 \mathrm{MW}$ y el Tipo 3 de 30MW. Como valor de inversión en generación se consideró un costo de 1 Millón\$/MW. Mientras que para el valor de inversión de líneas de transmisión se consideraron dos escenarios: precios altos y bajos. Para el escenario de precios de transmisión altos se consideraron los costos de líneas presentados en (Romero et al., 2005) y para el escenario de precios de transmisión bajos se consideraron los costos de líneas presentados en (Alizadeh y Jadid, 2011). En la Fig. 2 se presenta uno de los planes encontrados para el escenario de precios altos. Los elementos marcados en línea discontinua indican los nuevos elementos que propone el plan de expansión. En la Fig. 3 se presentan los frentes óptimos de Pareto para ambos escenarios de precios. Los tiempos de cálculo fueron 9541 y 10843 segundos para los escenarios de costos altos y bajos, respectivamente. Para ajustar los parámetros del NSGA-II se realizaron diferentes pruebas variando los parámetros del algoritmo. La combinación de parámetros que presenta los mejores resultados tras el ajuste de los mismos son: i) población=60; ii) generaciones=100; iii) tasa de cruce=0.9; y iv) tasa de mutación=0.1.

Las soluciones obtenidas con el algoritmo propuesto corresponden al conjunto no dominado (frente de Pareto). Es decir, desde el punto de vista de ambas funciones objetivo todas las soluciones son de la misma calidad. Cada una de estas representa un compromiso entre dos objetivos que están en conflicto (a mayor seguridad, mayor costo). Dentro del conjunto de soluciones del frente de Pareto no se puede afirmar que existe una solución mejor que otra. Ahora bien, es posible identificar la inversión mínima que garantice cierto nivel de seguridad. Desde el punto de vista del planeador de red, esta información es valiosa, pues puede utilizar los recursos disponibles reforzando los activos que mayor impacto tienen en la seguridad del sistema.

Los frentes de Pareto ilustrados en la Fig. 3 evidencian que una disminución en el costo de inversión implica un aumento en el índice nodal WTLR y viceversa. En la Fig. 3(a) se puede observar que, para garantizar que no haya sobrecargas, en operación normal ni bajo contingencias, se debe invertir en el plan de expansión al menos $1200 \mathrm{M} \$$. Inversiones mayores a este valor no contribuyen notoriamente a una reducción adicional de los índices WTLR. Por otro lado, inversiones menores a este valor no garantizan la seguridad del sistema, por lo que en algunas condiciones se podrían presentar sobrecargas que superen los límites permitidos. En la Fig. 3(b) se puede observar que las inversiones de al menos 750 M garantizan que el sistema no presenta sobrecargas que superen los límites permitidos en condición normal del sistema y ante contingencias. Inversiones mayores a $750 \mathrm{M} \$$ no contribuyen significativamente a un aumento de la seguridad; por otro lado, inversiones de menor valor disminuyen la seguridad del sistema.

En la Tabla 1 y en la Fig 2 se ilustra el plan de expansión más económico que garantiza índices WTRL cecanos a cero, considerando un escenario de precios altos en los activos de transmisión. En la primera columna de la Tabla 1 se presentan las líneas seleccionadas en el plan, mientras que la segunda columna presenta los nuevos generadores. La etiqueta Nodo (MW) indica la ubicación y potencia del generador propuesto como solución. Por ejemplo N1 (30) significa que un generador de $30 \mathrm{MW}$ será instalado en el nodo 1. La solución encontrada recomienda 21 líneas y 11 generadores con una potencia instalada de $190 \mathrm{MW}$. 


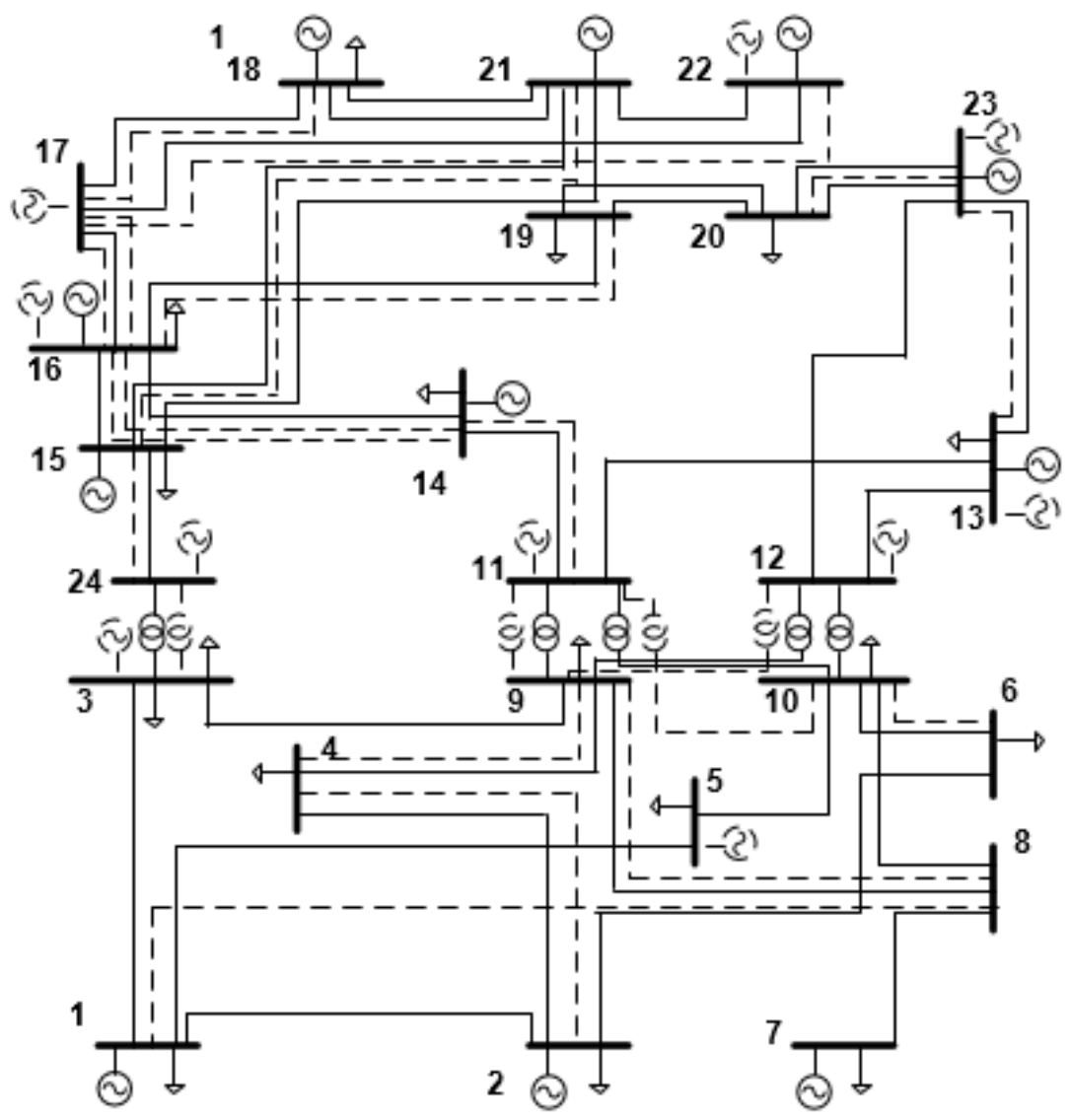

Fig. 2: Ilustración de un plan de expansión para el sistema IEEE RTS-24 (escenario precios altos)
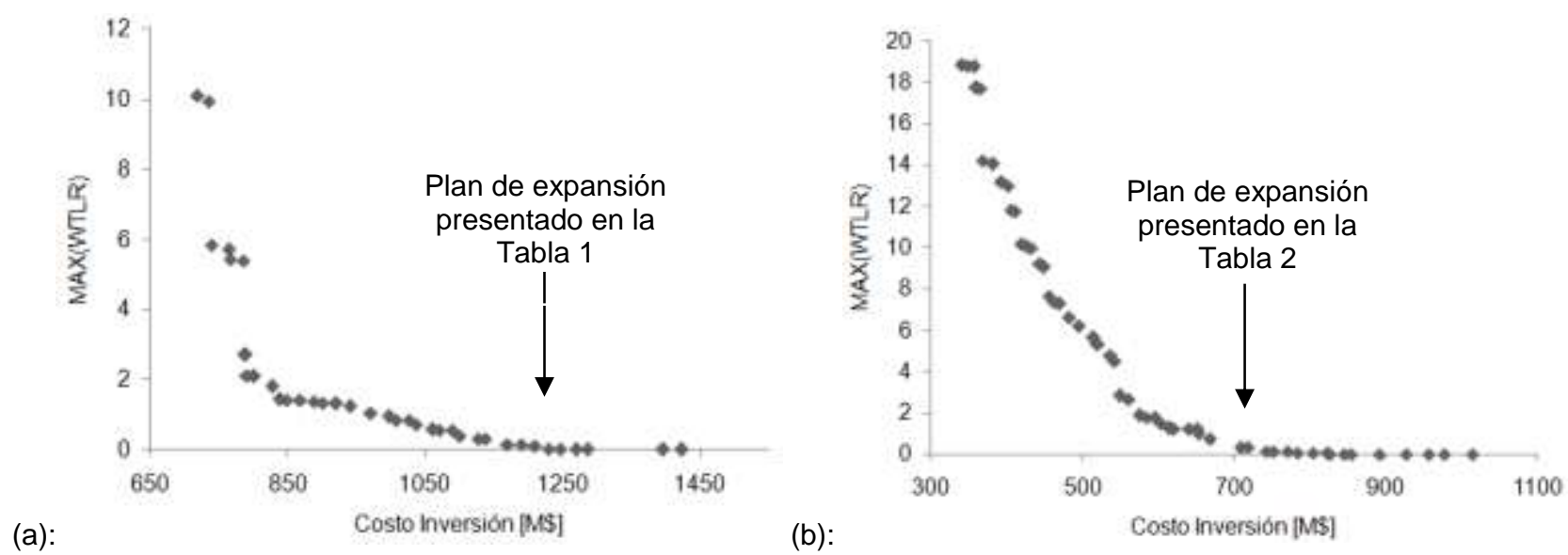

Fig. 3: Frente de Pareto. (a): escenario de precios altos, (b): escenario de precios bajos

En la Tabla se presenta el plan de expansión más económico en el escenario de precios bajos que no presentaría sobrecargas que superen los límites permitidos tanto en condición normal del sistema como ante contingencias (WTRL aproximadamente igual a cero). El plan recomienda 28 líneas y 10 generadores con una potencia instalada de 140MW. En este caso, el plan considera 7 líneas más que el escenario de precios altos.

Con el objetivo de verificar si los resultados del modelo PIEGT son mejores que los resultados del modelo PET tradicional, se resolvió el problema considerando solo líneas de transmisión como candidatos de expansión (en este caso se considera generación suficiente para atender la demanda). En la Fig. 4 se presenta el frente óptimo de Pareto para el PET considerando el escenario de precios altos. El tiempo de cálculo en este caso fue de 8289 segundos. Al comparar la Fig. 4 con la Fig.3(a) se puede observar que para garantizar altos niveles de seguridad (WTLR aproximadamente igual a cero) el PET requiere inversiones superiores al PIEGT. 
Tabla 1: Plan de expansión en transmisión y generación (escenario de precios altos)

\begin{tabular}{|c|c|c|c|}
\hline Líneas de transmisión & Generadores Nodo (MW) & $\begin{array}{c}\text { FO1 } \\
([\mathrm{M} \$])\end{array}$ & $\begin{array}{c}\text { FO2 } \\
\text { Max(WTRL) }\end{array}$ \\
\hline L3-24,L6-10,L9-11,L9-12,L14-16,L16- & N3(20),N5(10),N11(10),N11(30), & & \\
17,L17-18,L17-22,L1-8,L2-4,L4-9,L8- & N12(30),N13(30),N16(10), & 1270 & $1.74 \mathrm{E}-12$ \\
9,L10-11,L11-14,L13-23,L14-16,L15- & N17(10),N22(10),N23(10), & & \\
21,L15-24,L16-17,L16-19,L20-23 & N24(20), & & \\
\hline
\end{tabular}

Tabla 2: Plan de expansión en transmisión y generación (escenario de precios bajos)

\begin{tabular}{|c|c|c|c|}
\hline Líneas de transmisión & Generadores (MW) & $\begin{array}{c}\text { FO1 } \\
([\mathrm{M} \$])\end{array}$ & $\begin{array}{c}\text { FO2 } \\
\operatorname{Max}(\mathrm{WTRL}) \\
\end{array}$ \\
\hline $\begin{array}{l}\text { L2-6,L3-24,L4-9,L5-10,L6-10,L7-8,L8- } \\
\text { 9,L9-11,L10-12,L14-16,L15-21,L21- } \\
\text { 22,L14-23,L1-2,L1-3,L1-5,L3-9,L3-24,L6- } \\
\text { 10,L11-14,L15-21,L15-24,L16-17,L17- } \\
\text { 18,L18-21,L20-23,L1-8,L13-14 }\end{array}$ & $\begin{array}{c}\mathrm{N} 2(10), \mathrm{N} 3(20), \mathrm{N} 4(30), \mathrm{N} 5(20), \\
\mathrm{N} 6(10), \mathrm{N} 7(20), \mathrm{N} 9(10), \\
\mathrm{N} 18(10), \mathrm{N} 20(10), \mathrm{N} 22(10)\end{array}$ & 892 & $2 E-13$ \\
\hline
\end{tabular}

En la Tabla 4 se presentan los resultados del plan de expansión de mínimo costo que garantiza máxima seguridad. En este caso se contempla la instalación de 36 líneas (15 líneas más que las reportadas por el PIEGT para el mismo caso). Al comparar los resultados de la Tabla 3 con los resultados de la Tabla 1 se evidencia que para un nivel de seguridad dado el PIEGT puede encontrar soluciones más económicas que el PET. Las diferencias de resultados entre los modelos PIEGT y PET dependen de los costos de líneas y generadores que se incorporen a la red. La principal ventaja del PIEGT consiste en que la incorporación de generación en nodos estratégicos puede aplazar o sustituir parte de la expansión de la red de transmisión y aumentar la seguridad del sistema al suplir la demanda de forma local.

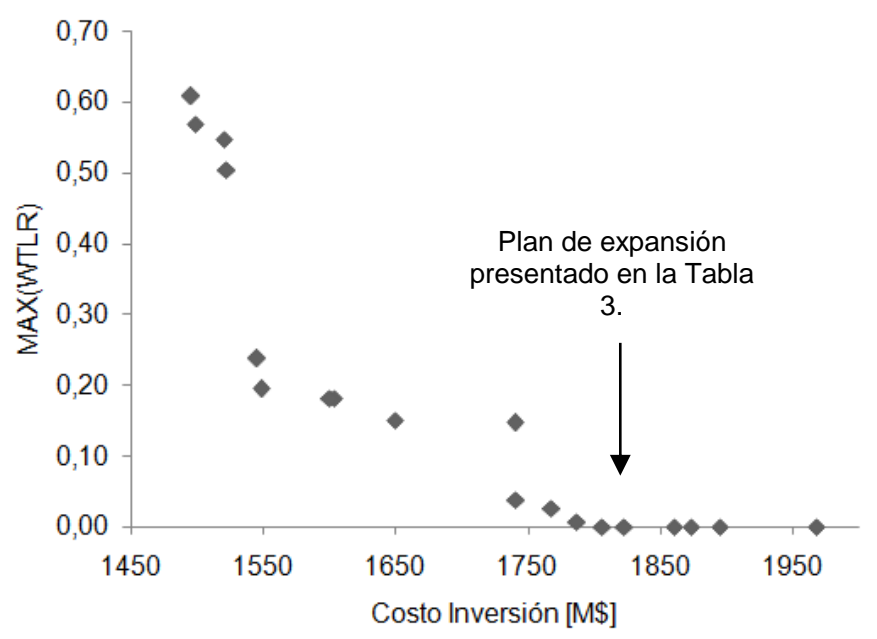

Fig. 4: Frente de Pareto del PET

Tabla 3: Plan de expansión en transmisión (escenario de precios altos)

\begin{tabular}{|c|c|c|}
\hline Líneas de transmisión & FO1 (C.I.[M\$]) & $\begin{array}{c}\mathrm{FO} 2 \\
\operatorname{Max}(\mathrm{WTRL})\end{array}$ \\
\hline $\begin{array}{l}\text { L1-5,L2-6,L3-24,L4-9,L5-10,L9-11,L10-12,L11-13,L14-16,L15-21,L16-17,L17- } \\
\text { 18,L17-22,L21-22,L13-14,L14-23,L1-5,L2-4,L2-6,L3-9,L3-24,L5-10,L6-10,L8- } \\
\text { 9,L8-10,L9-11,L11-14,L14-16,L15-16,L15-24,L16-17,L16-19,L20-23,L1-8,L14- } \\
\text { 23,L16-23 }\end{array}$ & 1806 & $1.15 \mathrm{E}-12$ \\
\hline
\end{tabular}

\section{CONCLUSIONES}

En este artículo se presentó un modelo de optimización y un método de solución para el PIEGT que busca minimizar los costos de inversión y maximizar la seguridad del sistema. El principal aporte de este artículo radica en la utilización de los índices nodales WTLR (expresados en función de los factores de distribución de potencia), los cuales no han sido utilizados previamente en modelos de optimización para el PIEGT. Los índices nodales WTLR no solo indican el nivel de seguridad del sistema, sino que identifican los nodos más 
sensibles a inyecciones de potencia. En este artículo los índices WTLR fueron utilizados con la doble función de diagnosticar el sistema en términos de congestión y guiar el algoritmo genético a encontrar mejores propuestas de expansión.

Abordar de forma integrada el planeamiento de la expansión en generación y transmisión permite explorar un conjunto de alternativas mucho mayor al que se tiene acceso cuando se abordan estos problemas por separado, o de forma secuencial. Se verificó que al incluir generación como alternativa de expansión, el número de líneas de transmisión necesarias para atender la demanda futura con altos niveles de seguridad se reduce considerablemente. Una ventaja adicional del método de solución propuesto radica en que no se obtiene como resultado un único plan de expansión, sino un conjunto de planes para diferentes niveles de seguridad deseados. Por lo tanto, de acuerdo a los requerimientos regulatorios y a los recursos económicos disponibles, el planeador podrá seleccionar un plan que se ajuste a sus necesidades.

\section{NOTACIÓN}

Conjuntos:

$\Omega_{b}$ : Conjunto de barras.

$\Omega_{l}$ : Conjunto de líneas existentes.

$\Omega_{g}$ : Conjunto de generadores existentes.

$\Omega_{l n}$ : Conjunto de líneas nuevas.

$\Omega_{g n}$ : Conjunto de generadores nuevos.

$\Omega_{c}$ : Conjunto de contingencias.

Parámetros:

$d_{i}$ : Demanda en la barra $i$ [MW].

$\bar{g}_{k}$ : Generación máxima del generador $k[\mathrm{MW}]$.

$c_{l}$ : Costo de inversión de la línea $l[\$]$.

$c_{k}$ : Costo de inversión del generador $k[\$]$.

$c o_{k}$ : Costo de operación del generador $k[\$ / M W]$.

$\bar{f}_{l}$ : Flujo de potencia máximo en la línea $l[\mathrm{MW}]$.

$x_{l}^{p u}:$ Reactancia de la línea $l$ [p.u].

$S_{\text {base }}$ : Potencia Base [MW].

$\bar{\theta}$ : Ángulo de fase máximo [rad].

$C_{D N A}$ : Costo de la demanda no atendida $[\$ / \mathrm{MW}]$.

Variables:

$w_{l}$ : Línea nueva $l$.

$z_{k}$ : Generador nuevo $k$.

$D N A_{i}$ : Demanda no atendida en el nodo $i[\mathrm{MW}]$.

$g_{k i}$ : Generación de potencia activa del generador $k$ conectado al nodo $i$ [MW].

$\theta_{i}$ : Ángulo de fase en la barra $i$ [rad].

$W T L R_{i}$ : Índice WTLR para el nodo $i$.

Nviol: Número de sobrecargas en operación normal y bajo contingencias.

sobrecarga $_{\text {sys }}$ : Sumatoria de todas las sobrecargas del sistema en operación normal y bajo contingencias $\mathrm{PCO}_{l}$ : Sobrecarga de la línea $l$ en condición normal de operación [MW].

$P C O_{l, c}$ : Sobrecarga en la línea $l$ ante contingencia de la línea $c[\mathrm{MW}]$.

$f_{l i j}$ : Flujo de potencia por la línea $l$ conectada entre los nodos $i, j$ en condición normal de operación [MW].

$f_{l i j, c}$ : Flujo de potencia por la línea $l$ conectada entre los nodos $i, j$ ante la contingencia $c$ [MW].

$I S F_{l}{ }^{i}$ : Sensibilidad del cambio de flujo de carga en la línea $l$ con respecto a una inyección de potencia en el nodo $i$ en condiciones normales de operación.

$I S F_{l, c}^{i}$ : Sensibilidad del cambio de flujo de carga en la línea $l$ con respecto a una inyección de potencia en el nodo $i$ ante contingencia de la línea $c$.

$L O D F_{l, c}$ : Sensibilidad del cambio del flujo de potencia de la línea $l$ ante la contingencia $c$.

\section{REFERENCIAS}

Aghaei, J., Akbari, M. A., Roosta, A. y Baharvandi, A., Multiobjective generation expansion planning considering power system adequacy, Electric Power Systems Research: 102(1), 8-19 (2013)

Alizadeh, B. y Jadid, S., Reliability constrained coordination of generation and transmission expansion planning in power systems using mixed integer programming, IET Generation, Transmission \& Distribution: 5(9), 948-960 (2011) 
Deb, K., Pratap, A., Agarwal, S. y Meyarivan., T., A fast and elitist multiobjective genetic algorithm: NSGA-II, IEEE Transactions on Evolutionary Computation:6(2), 182-197(2002)

Grigg, C., Wong, P., y otros 16 autores. The IEEE reliability test system -1996 a report prepared by the reliability test system task force of the application of probability methods subcommittee, IEEE Transactions on Power system: 14(3), 1010-1020 (1999)

Grijalva, S. y Visnesky, A. M., The effect of generation on network security: Spatial representation, metrics, and policy, IEEE Transactions on Power system:21(3), 1388-1395 (2006)

Güler, T., Gross, G. y Liu, M., Generalized line outage distribution factors, IEEE Transactions on Power system:22(2), 879-881 (2007)

Hemmati, R. y Hooshmand, R. A., State-of-the-art of transmission expansion planning: Comprehensive review, Renewable and Sustainable Energy Reviews:23(1), 312-319 (2013)

Javadi, M. S., Saniei, M., Mashhadi, H. R., y Gutiérrez, G. Multi-objective expansion planning approach: distant wind farms and limited energy resources integration, IET Renewable Power Generation: 7(6), 652668 (2013)

Jenabi, M., Fatemi, S. M. T y Smeers, Y., Bi-level game approaches for coordination of generation and transmission expansion planning within a market environment, IEEE Transactions on Power systems :28(3), 2639-2650 (2013)

Jin, S. y Ryan, S. M., A tri-level model of centralized transmission and decentralized generation expansion planning for an electricity market-Part II, IEEE Transactions on Power system: 29(1), 142-148 (2014)

Ma, X. y Zhou, Y., Coordination of Generation and Transmission Planning for Power System with Large Wind Farms, Energy Procedia:16(1), 1979-1985 (2012)

Motamedi, A., Zareipour, H., Buygi, M. O. y Rosehart, W. D., A transmission planning framework considering future generation expansions in electricity markets, IEEE Transactions on Power system: 25(4), 1987-1995 (2010)

Phupha, V., Lantharthong, T., Generation Expansion Planning Strategies on Power System: A Review, World Academy of Science Engineering and Tecnology:6(4), 436-439 (2012)

Pozo, D., Sauma, E. y Contreras, J., A Three-Level Static MILP Model for Generation and Transmission Expansion Planning, IEEE Transactions on Power system:28(1),202-210 (2013)

Quintero, J., Zhang, H., Chakhchoukh, Y.,Vittal,V. y Heydt, G.T., Next Generation Transmission Expansion Planning Framework: Models, Tools, and Educational Opportunities, IEEE Transactions on Power system:29(4), 911-1918, (2014)

Rojas-Rios, L.F., Lopez-Lezama J.M. y Muñoz-Galeano N., Asignación óptima de presupuesto para mejoramiento de la calidad del servicio en sistemas de distribucion usando NSGA-Il y un Algoritmo Memético, Revista Información Tecnológica: 27(1), 115-126, (2016)

Romero, R., Monticelli, A., Garcia, V. A. y Haffner, S., Test systems and mathematical models for transmission network expansion planning, IEE Proceedings on Generation, Transmission and Distribution:149(1), 27-36 (2002)

Romero, R., Rocha, C., Mantovani, y J.R., Sanchez, I.G., Constructive heuristic algorthm for the DC model in network transmission expansion planning, IEE Proceedings on Generation, Transmission and Distribution: 152(2) 277-281 (2005)

Rouhani, A., Hosseini, S. H. y Raoofat, M., Composite generation and transmission expansion planning considering distributed generation, Int. J. Electrical Power \& Energy Systems:62(1), 792-805 (2014)

Sharan, I. y Balasubramanian, R., Integrated generation and transmission expansion planning including power and fuel transportation constraints, Energy Policy:43(1), 275-284 (2012)

Srinivas, N. y Deb, K., Multiobjective Optimization Using Nondominated Sorting in Genetic Algorithms, Evolutionary Computation 2:3(1), 221-248 (1994)

Suarez, V.F., Guerrero, A. y Castrillón O.D., Programación de horarios escolares basados en ritmos cognitivos usando un algoritmo genético de clasificaion no dominado NSGA-II, Revista Informacion Tecnológica: 24(1), 103-114, (2013)

Ugranli, F. y Karatepe, E., Multi-objective transmission expansion planning considering minimization of curtailed wind energy, International Journal of Electrical Power \& Energy Systems: 65(1), 348-356 (2015) 